\title{
Architecture and function of human uromodulin filaments in urinary tract infections
}

\author{
Gregor L. Weiss ${ }^{1 *}$, Jessica J. Stanisich1*, Maximilian M. Sauer ${ }^{1 *+}$, Chia-Wei Lin ${ }^{2}$., Jonathan Eras ${ }^{1}$, Dawid S. \\ Zyla $^{1}$, Johannes Trück ${ }^{3}$, Olivier Devuyst ${ }^{4,5}$, Markus Aebi ${ }^{2}$, Martin Pilhofer ${ }^{1}$, Rudi Glockshuber ${ }^{1}$
}

${ }^{1}$ Institute of Molecular Biology and Biophysics, ETH Zürich, Otto-Stern-Weg 5, CH-8093 Zürich, Switzerland. ${ }^{2}$ Institute of Microbiology, ETH Zürich, Vladimir-Prelog-Weg 15/10, CH-8093 Zürich, Switzerland. ${ }^{3}$ University Children's Hospital Zürich, Steinwiesstrasse 75, CH-8032 Zürich, Switzerland. ${ }^{4}$ Institute of Physiology, Mechanisms of Inherited Kidney Disorders, University of Zürich, Winterthurerstrasse 190, CH-8057 Zürich, Switzerland. ${ }^{5}$ Division of Nephrology, UCLouvain Medical School, Brussels, Belgium.

*These authors contributed equally to this work. †Present address: Department of Biochemistry, University of Washington, 1705 NE Pacific St., Seattle, WA 98195, USA. ¥Present address: Functional Genomics Center Zürich, Winterthurerstrasse 190, CH-8057 Zürich, Switzerland.

§Corresponding author. Email: pilhofer@biol.ethz.ch

Uromodulin is the most abundant protein in human urine and forms filaments that antagonize the adhesion of uropathogens; however, filament structure and mechanism of protection remain poorly understood. We used cryo-electron tomography to show that the uromodulin filament consists of a zigzag-shaped backbone with laterally protruding arms. $\mathrm{N}$-glycosylation mapping and biophysical assays revealed that uromodulin acts as a multivalent ligand for the bacterial type 1 pilus adhesin, presenting specific epitopes on the regularly spaced arms. Imaging of uromodulin-uropathogen interactions in vitro and in patient urine showed that uromodulin filaments associate with uropathogens and mediate bacterial aggregation, which likely prevents adhesion and allows clearance by micturition. These results provide a framework for understanding uromodulin in urinary tract infections and in its more enigmatic roles in physiology and disease.

The glycoprotein Uromodulin (Umod) is secreted in the kidney and is the most abundant urinary protein (1). A $U M O D$ promoter variant in $\sim 80 \%$ of the human population drives a two-fold increase of urinary Umod levels (2), resulting in reduced susceptibility to bacterial urinary tract infections (UTIs) (3). Uropathogenic Escherichia coli (UPEC) utilize adhesive type 1 pili to attach to high-mannose type Nglycans displayed on the uroepithelial surface (4). Umod has been suggested to act as a soluble adhesion antagonist for UPECs (5-11).

Mature Umod consists of three epidermal growth factorlike domains (EGF I-III), a cysteine-rich domain (D8C), a fourth EGF domain (EGF IV) and the bipartite zona pellucida module (subdomains ZP-N and ZP-C) (Fig. 1A) (1). Produced as a glycosylphosphatidylinositol-anchored precursor, Umod is then cleaved by the protease hepsin and assembles into homopolymeric filaments with an average length of $\sim 2.5 \mu \mathrm{m}$ (12, 13). Despite its multiple roles in human health and disease (1), the molecular architecture and interactions of Umod in urine are poorly understood.

We first developed a protocol to purify stable Umod filaments from urine (table S1 and fig. S1A). Mass spectrometry (MS) of dissociated Umod monomers from different donors revealed highly similar, broad mass distribution profiles (fig. S1, B to D), showing that Umod glycosylation was gender- and genotype-independent. Next, we established a site-specific N- glycosylation map of Umod by LC-MS/MS analysis of tryptic glycopeptides (Fig. 1A, figs. S2 to S6, and tables S2 and S3). We identified individual $\mathrm{N}$-glycans attached to asparagine (Asn) residues 38, 76, 80, 232, 275, 322, 396 and 513 (figs. S2 to S6 and tables S2 and S3). High-mannose type N-glycans were found at Asn275 and Asn513, while other N-glycans were confirmed to be di-, tri-, or tetra-antennary complex types. Because UPEC adhere to uroepithelial cells via type 1 pili that specifically recognize high-mannose type N-glycans $(4,14)$, both glycans at Asn275 and Asn513 were candidates for mediating Umod's antiadhesive activity.

To contextualize the glycan arrangement within Umod polymers, we imaged Umod filaments by cryo-electron tomography (cryoET) (15) (Fig. 1B and movie S1). One orientation (Fig. 1C) was consistent with the previously observed zigzag shape $(12,13,16,17)$, though filaments showed different degrees of curvature and irregular rotations around the long axis. A second, prominent orientation had a fishbonelike appearance, with a central core filament and regularly protruding "arms" (Fig. 1D). We calculated a structure by subtomogram averaging, which resolved repeating filament-subunits arranged in a helix with $180^{\circ}$ twist and $6.5 \mathrm{~nm}$ rise. The filament core consisted of $8.5 \mathrm{~nm}$ long modules zigzagging at $95^{\circ}$ angles. The $12.5 \mathrm{~nm}$ long arm segments were flexible and protruded at $45^{\circ}$ angles (Fig. $1 \mathrm{E}$, fig. S7, and movie S1).

To assign Umod protein domains to densities in the 
subtomogram average, we studied an elastase-digested form of Umod (eUmod), wherein Umod is cleaved between the D8C and EGF IV domains (18). eUmod retained the zigzag core structure, while densities for the arms were absent (Fig. 1F; fig. S7; fig. S8, B to D; fig. S9; and movie S2). Based on the fitting of the previously published crystal structure of truncated Umod (EGF IV/ZP-module) (16) into our subtomogram averages, we propose a model in which the ZP module polymerizes into the filament backbone, while the EGF I-III and D8C domains constitute the protruding arms (Fig. 1G and fig. S9, C and D). Our "alternating ZP-stacking" model differs from previously suggested architectures $(16,19)$ of the Umod filament (fig. S10).

Next, we investigated the interaction between Umod filaments and the adhesin, FimH, of type 1-piliated UPEC strains. FimH at the pilus tip recognizes terminal mannosides in high-mannose type $\mathrm{N}$-glycans of the uroepithelial receptor uroplakin $1 \mathrm{a}(4,20)$. Binding of type 1-piliated cells to Umod filaments had been demonstrated, but the exact FimH binding site in Umod remained unknown (6). We recorded the affinity of the isolated FimH lectin domain $\left(\mathrm{FimH}_{\mathrm{L}}\right)$ to Umod filaments and obtained a single apparent dissociation constant of $2.2 \times 10^{-8} \mathrm{M}$ (Fig. 2A). Consistent with the high affinity of $\mathrm{FimH}_{\mathrm{L}}$ for Umod, spontaneous dissociation of $\mathrm{FimH}_{\mathrm{L}}$ from Umod filaments was very slow, with a half-life of 2.1 hours (Fig. 2B). As FimH $\mathrm{L}_{\mathrm{L}}$ binds mannosides with a 2000-fold higher affinity than full-length FimH as a consequence of the ability of FimH to form catch bonds under tensile mechanical force $(21,22)$, we calculated a $\mathrm{K}_{\mathrm{d}}$ of $\sim 4 \times 10^{-5} \mathrm{M}$ for the Umod/FimH complex in the absence of shear stress. Using analytical gel filtration and gel band densitometry, we determined the stoichiometry of the Umod/FimH $\mathrm{L}_{\mathrm{L}}$ complex to be 1:2 (Fig. 2, C and D, and fig. S11).

To test which of the two high-mannose type N-glycans of Umod was recognized by FimH, we analyzed the Umod.(FimH $\left.\mathrm{L}_{\mathrm{L}}\right)_{2}$ complex by cryoET. Differences between $\mathrm{Umod} \cdot\left(\mathrm{FimH}_{\mathrm{L}}\right)_{2}$ and native Umod were already visible in individual tomograms (fig. S12, A and B, and movie S3). The Umod. $\left(\text { FimH } \mathrm{L}_{\mathrm{L}}\right)_{2}$ subtomogram average revealed a prominent additional density on the Umod arms, which may be sufficient to accommodate two FimH $_{\mathrm{L}}$ (Fig. 2, E and F; fig. S12, C to F; and movie S3). No significant additional density was seen at the core of Umod $\left(\mathrm{FimH}_{\mathrm{L}}\right)_{2}$ filaments or on eUmod (contains only the high mannose-glycan at Asn513) incubated with $\mathrm{FimH}_{\mathrm{L}}$ (fig. S12, G to L). Together, our data demonstrate that the Man5-9 glycan at Asn275 of the Umod arm is the only accessible $\mathrm{FimH}_{\mathrm{L}}$ recognition site.

We next analyzed binding of Umod filaments to a mixture of type 1-piliated and non-piliated $E$. coli cells by cryoET imaging. Each type 1-piliated cell exhibited a significant increase in the local Umod filament concentration around the cells ( $n=6$ tomograms) (Fig. $3, \mathrm{~A}$ and B; fig. S13, A and B; and movie S4). Contact sites between pili and Umod were mainly at the FimH-containing pilus tips (Fig. 3A). In contrast, no Umod filaments accumulated around non-piliated cells ( $n=7$ tomograms; fig. S13, C and D) or piliated cells incubated with eUmod ( $n=3$ tomograms; fig. S14). E. coliUmod association therefore requires both the presence of type 1 pili and the glycosylated Umod arm.

Using light microscopy, we investigated bacteria-Umod interactions on a larger scale. Incubation of type 1-piliated $E$. coli with Umod filaments resulted in the aggregation of bacteria and the formation of clumps consisting of tens to hundreds of bacteria (Fig. 3C). Cell clumping occurred over a wide range of Umod concentrations and was inhibited by an excess of D-mannose (Fig. 3C), indicating that clumping was caused by FimH binding to Umod glycans. Pre-formed clumps, however, proved to be resistant against dissociation by D-mannose (fig. S15). TIRF microscopy showed that cell aggregates co-localized with Umod (Fig. 3D). Umod-dependent cell aggregation also occurred with a UPEC strain (fig. S16), but was not detected with non-piliated E. coli or with piliated E. coli incubated with eUmod (figs. S17 and S18). To visualize the Umod-induced cell aggregates by cryoET, we thinned the sample using cryo-focused ion beam (FIB) milling (23). The cryotomograms consistently revealed bacteria that were tightly surrounded by a dense mesh of Umod filaments ( $n=17$ tomograms; Fig. 3E, fig. S19, and movie S5).

In addition to type 1 pili, many UPEC strains express multiple pili with diverse glycan specificities. The UPEC strain CFT073 encodes type 1 pili (mannoside specific lectin), F9/Fml pili [lectin specific for galactosides], and S-pili [lectin specific for sialic acid] (24-26). We therefore tested the inhibition of Umod-mediated cell clumping of CFT073 by the addition of D-mannose, D-galactose and sialic acid. While Dmannose alone only slightly decreased cell aggregation (fig. S20, A and B), the addition of a cocktail of all three monosaccharides proved to be most effective in reducing Umod-mediated cell clumping (fig. S20B). This indicates that the complex-type Umod glycans might interact with different types of pilus adhesins presented by different uropathogens.

We verified these findings by analyzing unprocessed urine from patients with clinically diagnosed UTIs by light microscopy and cryoET. Urine from a patient with an E. coli UTI revealed bacterial clumps that were embedded into fibrous structures (Fig. 4A). Individual bacteria of the same sample were heavily piliated and always surrounded by numerous Umod filaments (Fig. 4B and movie S6; $n=27$ tomograms); several contact sites between pilus tips and Umod were resolved (Fig. 4C and movie S6). Furthermore, we analyzed urine from UTI patients with other pathogens, including Klebsiella pneumoniae, Pseudomonas aeruginosa and Streptococcus mitis. In all cases, we observed cell aggregates associated with fibrous structures in light microscopy, and 67- 
$71 \%$ of cells imaged by cryoET confirmed the presence of Umod filaments (fig. S21 and movie S7).

In conclusion, our data provide a 3D structure of native Umod filaments and support the hypothesis that the polymerization of Umod into a multivalent filament is required for its function as a FimH antagonist to effectively compete with the high concentration of uroplakin la on the urinary epithelium $(4,14,27)$. The intrinsic flexibility of Umod filaments allows their adaptation to the bacterial surface, multivalent binding to pili from the same bacterium and eventually encapsulation of entire cells. Analogous to the mechanism by which antibody-crosslinked clumps of enchained Salmonella cells facilitates the removal of pathogens from the gut (28), Umod-mediated cell aggregation may have a role in efficient clearance of bacteria via micturition.

Our study also sheds light on interactions between Umod and pathogens other than type 1-piliated UPECs. UPEC genomes, for instance, encode up to 16 fimbrial gene clusters and UPEC switch expression between pilus types with distinct receptor specificities $(24,29-31)$. The seven different complex type $\mathrm{N}$-glycans on Umod (Fig. 1A) may therefore competitively inhibit adhesion of other pathogens to other epithelial receptors. Finally, the resolved site-specific N-glycosylation pattern and architecture of Umod filaments will serve as a framework for studying the mechanisms underlying Umod's roles in the regulation of salt transport, kidney diseases and innate immunity $(1,19)$.

\section{REFERENCES AND NOTES}

1. O. Devuyst, E. Olinger, L. Rampoldi, Uromodulin: From physiology to rare and complex kidney disorders. Nat. Rev. Nephrol. 13, 525-544 (2017). doi:10.1038/nrneph.2017.101 Medline

2. M. Trudu, S. Janas, C. Lanzani, H. Debaix, C. Schaeffer, M. Ikehata, L. Citterio, S. Demaretz, F. Trevisani, G. Ristagno, B. Glaudemans, K. Laghmani, G. Dell'Antonio, SKIPOGH team, J. Loffing, M. P. Rastaldi, P. Manunta, O. Devuyst, L. Rampoldi, Common noncoding UMOD gene variants induce salt-sensitive hypertension and kidney damage by increasing uromodulin expression. Nat. Med. 19, 1655-1660 (2013). doi:10.1038/nm.3384 Medline

3. S. Ghirotto, F. Tassi, G. Barbujani, L. Pattini, C. Hayward, P. Vollenweider, M. Bochud, L. Rampoldi, O. Devuyst, The Uromodulin Gene Locus Shows Evidence of Pathogen Adaptation through Human Evolution. J. Am. Soc. Nephrol. 27, 29832996 (2016). doi:10.1681/ASN.2015070830 Medline

4. G. Zhou, W.-J. Mo, P. Sebbel, G. Min, T. A. Neubert, R. Glockshuber, X.-R. Wu, T.-T. Sun, X.-P. Kong, Uroplakin la is the urothelial receptor for uropathogenic Escherichia coli: Evidence from in vitro FimH binding. J. Cell Sci. 114, 4095-4103 (2001). Medline

5. J. Parkkinen, R. Virkola, T. K. Korhonen, Identification of factors in human urine that inhibit the binding of Escherichia coli adhesins. Infect. Immun. 56, 2623-2630 (1988). doi:10.1128/lAl.56.10.2623-2630.1988 Medline

6. J. Pak, Y. Pu, Z. T. Zhang, D. L. Hasty, X. R. Wu, Tamm-Horsfall protein binds to type 1 fimbriated Escherichia coli and prevents E. coli from binding to uroplakin la and Ib receptors. J. Biol. Chem. 276, 9924-9930 (2001). doi:10.1074/jbc.M008610200 Medline

7. M. S. Conover, S. Ruer, J. Taganna, V. Kalas, H. De Greve, J. S. Pinkner, K. W. Dodson, H. Remaut, S. J. Hultgren, Inflammation-Induced Adhesin-Receptor Interaction Provides a Fitness Advantage to Uropathogenic E. coli during Chronic Infection. Cell Host Microbe 20, 482-492 (2016). doi:10.1016/i.chom.2016.08.013 Medline

8. F. Serafini-Cessi, A. Monti, D. Cavallone, N-Glycans carried by Tamm-Horsfall glycoprotein have a crucial role in the defense against urinary tract diseases. Glycoconj. J. 22, 383-394 (2005). doi:10.1007/s10719-005-2142-z Medline

9. D. Cavallone, N. Malagolini, A. Monti, X.-R. Wu, F. Serafini-Cessi, Variation of high mannose chains of Tamm-Horsfall glycoprotein confers differential binding to type 1-fimbriated Escherichia coli. J. Biol. Chem. 279, 216-222 (2004). doi:10.1074/ibc.M308821200 Medline

10. J. M. Bates Jr., H. M. Raffi, K. Prasadan, R. Mascarenhas, Z. Laszik, N. Maeda, S. J. Hultgren, S. Kumar, Tamm-Horsfall protein knockout mice are more prone to urinary tract infection: Rapid communication. Kidney Int. 65, 791-797 (2004). doi:10.1111/i.1523-1755.2004.00452.x Medline

11. L. Mo, X.-H. Zhu, H.-Y. Huang, E. Shapiro, D. L. Hasty, X.-R. Wu, Ablation of the Tamm-Horsfall protein gene increases susceptibility of mice to bladder colonization by type 1-fimbriated Escherichia coli. Am. J. Physiol. Renal Physiol. 286, F795-F802 (2004). doi:10.1152/ajprenal.00357.2003 Medline

12. M. Brunati, S. Perucca, L. Han, A. Cattaneo, F. Consolato, A. Andolfo, C. Schaeffer, E. Olinger, J. Peng, S. Santambrogio, R. Perrier, S. Li, M. Bokhove, A. Bachi, E. Hummler, O. Devuyst, Q. Wu, L. Jovine, L. Rampoldi, The serine protease hepsin mediates urinary secretion and polymerisation of Zona Pellucida domain protein uromodulin. eLife 4, e08887 (2015). doi:10.7554/eLife.08887 Medline

13. K. R. Porter, I. Tamm, Direct visualization of a mucoprotein component of urine. J. Biol. Chem. 212, 135-140 (1955). Medline

14. B. Xie, G. Zhou, S.-Y. Chan, E. Shapiro, X.-P. Kong, X.-R. Wu, T.-T. Sun, C. E. Costello, Distinct glycan structures of uroplakins la and lb: Structural basis for the selective binding of FimH adhesin to uroplakin la. J. Biol. Chem. 281, 14644-14653 (2006). doi:10.1074/jbc.M600877200 Medline

15. M. Beck, W. Baumeister, Cryo-Electron Tomography: Can it Reveal the Molecular Sociology of Cells in Atomic Detail? Trends Cell Biol. 26, 825-837 (2016). doi:10.1016/i.tcb.2016.08.006 Medline

16. M. Bokhove, K. Nishimura, M. Brunati, L. Han, D. de Sanctis, L. Rampoldi, L. Jovine, A structured interdomain linker directs self-polymerization of human uromodulin. Proc. Natl. Acad. Sci. U.S.A. 113, 1552-1557 (2016). doi:10.1073/pnas.1519803113 Medline

17. J. S. Hunt, P. R. MacDonald, W. A. Day, A. R. McGiven, Biochemical and mitogenic properties of Tamm-Horsfall urinary glycoprotein. Pathology 12, 609-621(1980). doi:10.3109/00313028009086814 Medline

18. L. Jovine, H. Qi, Z. Williams, E. Litscher, P. M. Wassarman, The ZP domain is a conserved module for polymerization of extracellular proteins. Nat. Cell Biol. 4 , 457-461 (2002). doi:10.1038/ncb802 Medline

19. L. Rampoldi, F. Scolari, A. Amoroso, G. Ghiggeri, O. Devuyst, The rediscovery of uromodulin (Tamm-Horsfall protein): From tubulointerstitial nephropathy to chronic kidney disease. Kidney Int. 80, 338-347 (2011). doi:10.1038/ki.2011.134 Medline

20. E. Hahn, P. Wild, U. Hermanns, P. Sebbel, R. Glockshuber, M. Häner, N. Taschner, P. Burkhard, U. Aebi, S. A. Müller, Exploring the 3D molecular architecture of Escherichia coli type 1 pili. J. Mol. Biol. 323, 845-857 (2002). doi:10.1016/S00222836(02)01005-7 Medline

21. M. M. Sauer, R. P. Jakob, J. Eras, S. Baday, D. Eriş, G. Navarra, S. Bernèche, B. Ernst, T. Maier, R. Glockshuber, Catch-bond mechanism of the bacterial adhesin FimH. Nat. Commun. 7, 10738 (2016). doi:10.1038/ncomms10738 Medline

22. M. M. Sauer, R. P. Jakob, T. Luber, F. Canonica, G. Navarra, B. Ernst, C. Unverzagt, T. Maier, R. Glockshuber, Binding of the Bacterial Adhesin FimH to Its Natural, Multivalent High-Mannose Type Glycan Targets. J. Am. Chem. Soc. 141, 936-944 (2019). doi:10.1021/jacs.8b10736 Medline

23. J. M. Medeiros, D. Böck, M. Pilhofer, Imaging bacteria inside their host by cryofocused ion beam milling and electron cryotomography. Curr. Opin. Microbiol. 43, 62-68 (2018). doi:10.1016/j.mib.2017.12.006 Medline

24. D. J. Wurpel, S. A. Beatson, M. Totsika, N. K. Petty, M. A. Schembri, Chaperoneusher fimbriae of Escherichia coli. PLOS ONE 8, e52835 (2013) doi:10.1371/journal.pone.0052835 Medline

25. D. J. Wurpel, M. Totsika, L. P. Allsopp, L. E. Hartley-Tassell, C. J. Day, K. M. Peters, S. Sarkar, G. C. Ulett, J. Yang, J. Tiralongo, R. A. Strugnell, M. P. Jennings, M. A. Schembri, F9 fimbriae of uropathogenic Escherichia coli are expressed at low temperature and recognise Galß1-3GIcNAc-containing glycans. PLOS ONE 9, e93177 (2014). doi:10.1371/journal.pone.0093177 Medline

26. T. J. Wiles, R. R. Kulesus, M. A. Mulvey, Origins and virulence mechanisms of 
uropathogenic Escherichia coli. Exp. Mol. Pathol. 85, 11-19 (2008). doi:10.1016/i.yexmp.2008.03.007 Medline

27. G. Min, M. Stolz, G. Zhou, F. Liang, P. Sebbel, D. Stoffler, R. Glockshuber, T.-T. Sun, U. Aebi, X.-P. Kong, Localization of uroplakin la, the urothelial receptor for bacterial adhesin FimH, on the six inner domains of the $16 \mathrm{~nm}$ urothelial plaque particle. J. Mol. Biol. 317, 697-706 (2002). doi:10.1006/imbi.2002.5442 Medline

28. K. Moor, M. Diard, M. E. Sellin, B. Felmy, S. Y. Wotzka, A. Toska, E. Bakkeren, M. Arnoldini, F. Bansept, A. D. Co, T. Völler, A. Minola, B. Fernandez-Rodriguez, G. Agatic, S. Barbieri, L. Piccoli, C. Casiraghi, D. Corti, A. Lanzavecchia, R. R. Regoes, C. Loverdo, R. Stocker, D. R. Brumley, W.-D. Hardt, E. Slack, High-avidity IgA protects the intestine by enchaining growing bacteria. Nature 544, 498-502 (2017). doi:10.1038/nature22058 Medline

29. N. Holden, M. Totsika, L. Dixon, K. Catherwood, D. L. Gally, Regulation of P-fimbrial phase variation frequencies in Escherichia coli CFT073. Infect. Immun. 75, 33253334 (2007). doi:10.1128/LAl.01989-06 Medline

30. S. Lindberg, Y. Xia, B. Sondén, M. Göransson, J. Hacker, B. E. Uhlin, Regulatory Interactions among adhesin gene systems of uropathogenic Escherichia coli. Infect. Immun. 76, 771-780 (2008). doi:10.1128/AAL.01010-07 Medline

31. Y. Xia, D. Gally, K. Forsman-Semb, B. E. Uhlin, Regulatory cross-talk between adhesin operons in Escherichia coli: Inhibition of type 1 fimbriae expression by the PapB protein. EMBO J. 19, 1450-1457 (2000). doi:10.1093/emboj/19.7.1450 Medline

32. D. Cavallone, N. Malagolini, G.-M. Frascà, S. Stefoni, F. Serafini-Cessi, Saltprecipitation method does not isolate to homogeneity Tamm-Horsfall glycoprotein from urine of proteinuric patients and pregnant women. Clin. Biochem. 35, 405-410 (2002). doi:10.1016/S0009-9120(02)00329-6 Medline

33. I. Hang, C.-W. Lin, O. C. Grant, S. Fleurkens, T. K. Villiger, M. Soos, M. Morbidelli, R. J. Woods, R. Gauss, M. Aebi, Analysis of site-specific N-glycan remodeling in the endoplasmic reticulum and the Golgi. Glycobiology 25, 1335-1349 (2015). doi:10.1093/glycob/cwv058 Medline

34. W. Colón, Analysis of protein structure by solution optical spectroscopy. Methods Enzymol. 309, 605-632 (1999). doi:10.1016/S0076-6879(99)09041-2 Medline

35. I. C. Blomfjeld, M. S. McClain, B. I. Eisenstein, Type 1 fimbriae mutants of Escherichia coli K12: Characterization of recognized afimbriate strains and construction of new fim deletion mutants. Mol. Microbiol. 5, 1439-1445 (1991). doi:10.1111/i.1365-2958.1991.tb00790.x Medline

36. P. E. Orndorff, S. Falkow, Identification and characterization of a gene product that regulates type 1 piliation in Escherichia coli. J. Bacteriol. 160, 61-66 (1984). doi:10.1128/JB.160.1.61-66.1984 Medline

37. G. L. Weiss, J. M. Medeiros, M. Pilhofer, In Situ Imaging of Bacterial Secretion Systems by Electron Cryotomography. Methods Mol. Biol. 1615, 353-375 (2017). doi:10.1007/978-1-4939-7033-9 27 Medline

38. W. F. Tivol, A. Briegel, G. J. Jensen, An improved cryogen for plunge freezing. Microsc. Microanal. 14, 375-379 (2008). doi:10.1017/S1431927608080781 Medline

39. C. V. lancu, W. F. Tivol, J. B. Schooler, D. P. Dias, G. P. Henderson, G. E. Murphy, E. R. Wright, Z. Li, Z. Yu, A. Briegel, L. Gan, Y. He, G. J. Jensen, Electron cryotomography sample preparation using the Vitrobot. Nat. Protoc. 1, 28132819 (2006). doi:10.1038/nprot.2006.432 Medline

40. M. Schorb, I. Haberbosch, W. J. H. Hagen, Y. Schwab, D. N. Mastronarde, Software tools for automated transmission electron microscopy. Nat. Methods 16, 471-477 (2019). doi:10.1038/s41592-019-0396-9 Medline

41. S. Q. Zheng, B. Keszthelyi, E. Branlund, J. M. Lyle, M. B. Braunfeld, J. W. Sedat, D. A. Agard, UCSF tomography: An integrated software suite for real-time electron microscopic tomographic data collection, alignment, and reconstruction. J. Struct. Biol. 157, 138-147 (2007). doi:10.1016/i.jsb.2006.06.005 Medline

42. D. N. Mastronarde, S. R. Held, Automated tilt series alignment and tomographic reconstruction in IMOD. J. Struct. Biol. 197, 102-113 (2017). doi:10.1016/i.jsb.2016.07.011 Medline

43. D. Tegunov, P. Cramer, Real-time cryo-electron microscopy data preprocessing with Warp. Nat. Methods 16, 1146-1152 (2019). doi:10.1038/s41592-019-0580-y Medline

44. D. Nicastro, C. Schwartz, J. Pierson, R. Gaudette, M. E. Porter, J. R. McIntosh, The molecular architecture of axonemes revealed by cryoelectron tomography. Science 313, 944-948 (2006). doi:10.1126/science.1128618 Medline
45. D. Castaño-Díez, M. Kudryashev, M. Arheit, H. Stahlberg, Dynamo: A flexible, userfriendly development tool for subtomogram averaging of cryo-EM data in highperformance computing environments. J. Struct. Biol. 178, 139-151 (2012). doi:10.1016/i.jsb.2011.12.017 Medline

46. E. F. Pettersen, T. D. Goddard, C. C. Huang, G. S. Couch, D. M. Greenblatt, E. C. Meng, T. E. Ferrin, UCSF Chimera-A visualization system for exploratory research and analysis. J. Comput. Chem. 25, 1605-1612 (2004). doi:10.1002/jcc.20084 Medline

47. J. M. Medeiros, D. Böck, G. L. Weiss, R. Kooger, R. A. Wepf, M. Pilhofer, Robust workflow and instrumentation for cryo-focused ion beam milling of samples for electron cryotomography. Ultramicroscopy 190, 1-11 (2018). doi:10.1016/i.ultramic.2018.04.002 Medline

48. M. Schaffer, B. D. Engel, T. Laugks, J. Mahamid, J. M. Plitzko, W. Baumeister, Cryofocused ion beam sample preparation for imaging vitreous cells by cryo-electron tomography. Bio Protoc. 5, e1575 (2015). doi:10.21769/bioprotoc. 1575 Medline

49. P. Kuzmič, Program DYNAFIT for the analysis of enzyme kinetic data: Application to HIV proteinase. Anal. Biochem. 237, 260-273 (1996). doi:10.1006/abio.1996.0238 Medline

50. F. P. Lindberg, B. Lund, S. Normark, Genes of pyelonephritogenic E. coli required for digalactoside-specific agglutination of human cells. EMBO J. 3, 1167-1173 (1984). doi:10.1002/j.1460-2075.1984.tb01946.x Medline

51. J. Schindelin, I. Arganda-Carreras, E. Frise, V. Kaynig, M. Longair, T. Pietzsch, S. Preibisch, C. Rueden, S. Saalfeld, B. Schmid, J.-Y. Tinevez, D. J. White, V. Hartenstein, K. Eliceiri, P. Tomancak, A. Cardona, Fiji: An open-source platform for biological-image analysis. Nat. Methods 9, 676-682 (2012). doi:10.1038/nmeth.2019 Medline

52. A. M. S. Grant, A. Neuberger, The turnover rate of rabbit urinary Tamm-Horsfall glycoprotein. Biochem. J. 136, 659-668 (1973). doi:10.1042/bj1360659 Medline

53. S. Youhanna, J. Weber, V. Beaujean, B. Glaudemans, J. Sobek, O. Devuyst, Determination of uromodulin in human urine: Influence of storage and processing. Nephrol. Dial. Transplant. 29, 136-145 (2014). doi:10.1093/ndt/gft345 Medline

54. K. Hård, G. Van Zadelhoff, P. Moonen, J. P. Kamerling, F. G. Vliegenthart, The Asnlinked carbohydrate chains of human Tamm-Horsfall glycoprotein of one male. Novel sulfated and novel $\mathrm{N}$-acetylgalactosamine-containing $\mathrm{N}$-linked carbohydrate chains. Eur. J. Biochem. 209, 895-915 (1992). doi:10.1111/i.14321033.1992.tb17362.x Medline

55. J. J. van Rooijen, A. F. Voskamp, J. P. Kamerling, J. F. Vliegenthart, Glycosylation sites and site-specific glycosylation in human Tamm-Horsfall glycoprotein. Glycobiology 9, 21-30 (1999). doi:10.1093/glycob/9.1.21 Medline

56. J. J. Van Rooijen, P. Hermentin, J. P. Kamerling, J. F. G. Vliegenthart, The patterns of the complex- and oligomannose-type glycans of uromodulin (Tamm-Horsfall glycoprotein) in the course of pregnancy. Glycoconj. J. 18, 539-546 (2001). doi:10.1023/A:1019644413639 Medline

57. P. Roepstorff, J. Fohlman, Proposal for a common nomenclature for sequence ions in mass spectra of peptides. Biomed. Mass Spectrom. 11, 601 (1984). doi:10.1002/bms.1200111109 Medline

58. A. Dell, A. J. Reason, K. H. Khoo, M. Panico, R. A. McDowell, H. R. Morris, Mass spectrometry of carbohydrate-containing biopolymers. Methods Enzymol. 230, 108-132 (1994). doi:10.1016/0076-6879(94)30010-0 Medline

59. C. Schaeffer, S. Santambrogio, S. Perucca, G. Casari, L. Rampoldi, Analysis of uromodulin polymerization provides new insights into the mechanisms regulating ZP domain-mediated protein assembly. Mol. Biol. Cell 20, 589-599 (2009). doi:10.1091/mbc.e08-08-0876 Medline

60. S. Toyota, Y. Fukushi, S. Katoh, S. Orikasa, Y. Suzuki, Anti-bacterial defense mechanism of the urinary bladder: Role of mannose in urine. Nippon Hinyokika Gakkai Zasshi 80, 1816-1823 (1989). doi:10.5980/ipnjurol1989.80.1816 Medline

61. C.-Y. Hong, M. Abdullah, N.-K. Wong, Evaluation of glycan profiles of TammHorsfall glycoprotein and uromodulin. Int. J. Pharm. Pharm. Sci. 5, 385-389 (2013).

62. S. Patel, N. Mathivanan, A. Goyal, Bacterial adhesins, the pathogenic weapons to trick host defense arsenal. Biomed. Pharmacother. 93, 763-771 (2017). doi:10.1016/i.biopha.2017.06.102 Medline

63. I. Margarit y Ros, in Streptococcus pyogenes: Basic Biology to Clinical Manifestations, J. J. Ferretti, D. L. Stevens, V. A. Fischetti, Eds. (Univ. of Oklahoma Health Sciences Center, 2016) 


\section{ACKNOWLEDGMENTS}

We thank the Functional Genomics Center Zürich, specifically S. Chesnov and P. Hunziker, for performing the MALDI-MS analysis and Edman sequencing, $\mathrm{H}$. Debaix for the genotyping, E. Olinger and A. Yoshifuji for support in urinary Umod analyses, P. Tittmann and M. Peterek for technical support during EM data collection and ScopeM for instrument access at ETH Zürich. F. Eisenstein is acknowledged for help with subtomogram average processing and J. Xu for help with analyzing cryoET data. We thank G. Navarra and B. Ernst (University of Basel) for providing the fluorescent mannoside GN-FP. C. Giese is acknowledged for providing E. coli AAEC189 [pSH2] cells. Funding: GLW was supported by a Boehringer Ingelheim Fonds PhD fellowship. MP was supported by the Swiss National Science Foundation (\#31003A_179255), the European Research Council (\#679209) and the NOMIS foundation. RG was supported by the Swiss National Science Foundation (310030B_176403/1 and 31003A_156304). OD was supported by the Swiss National Centre of Competence in Research Kidney Control of Homeostasis (NCCR Kidney.CH) program, the Swiss National Science Foundation (310030_189044) and the Rare Disease Initiative Zürich (Radiz). JT was supported by the Swiss National Science Foundation (PZ00P3_161147;

PZOOP3_183777). MP and RG were also supported by basic funding from ETH Zürich. Author contributions: G.L.W., J.J.S. and M.M.S. contributed equally. G.L.W., J.J.S., M.M.S., O.D., M.P. and R.G. designed experiments. J.J.S., M.M.S. and J.E. purified and biophysically analyzed Umod. C.-W.L. and M.A. performed and analyzed MS experiments. G.L.W. collected and processed cryoET data. D.S.Z. constructed the expression plasmid and performed TIRF microscopy. J.J.S. performed and analyzed light microscopy experiments. G.L.W. and J.T. collected patient urine. G.L.W., J.J.S, M.M.S., M.P. and R.G. wrote the manuscript with comments from all authors. Competing interests: The authors declare no competing interests. Data and materials availability: All subtomogram averages shown in this study were uploaded to the Electron Microscopy Databank (EMD) together with their respective half maps, masks for FSC calculations and an example tomogram. Accession numbers: EMD-11128/EMD11129 (native Umod), EMD-11130/EMD11131 (eUmod), EMD-1133/EMD-11134 (Umod incubated with FimH $\mathrm{L}_{\mathrm{L}}$ ) and EMD-11134/EMD-11135 (eUmod incubated with FimH $\mathrm{L}_{\mathrm{L}}$. Example tomograms of bacterial cells with Umod were also uploaded to the Electron Microscopy Databank with accession numbers EMD11136 to EMD-11143. All other data needed to evaluate the conclusions in the paper are present in the paper or the supplementary materials.

\section{SUPPLEMENTARY MATERIALS}

science.sciencemag.org/cgi/content/full/science.aaz9866/DC1

Materials and Methods

Figs. S1 to S21

Tables S1 to S4

References (32-63)

MDAR Reproducibility Checklist

Movies S1 to S7

25 October 2019; resubmitted 22 April 2020

Accepted 18 June 2020

Published online 2 July 2020

10.1126/science.aaz9866 
A

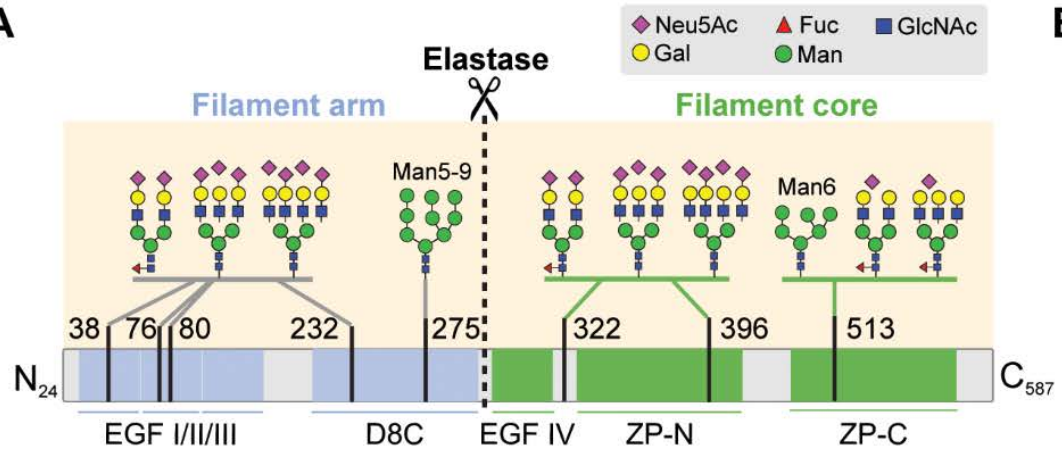

E

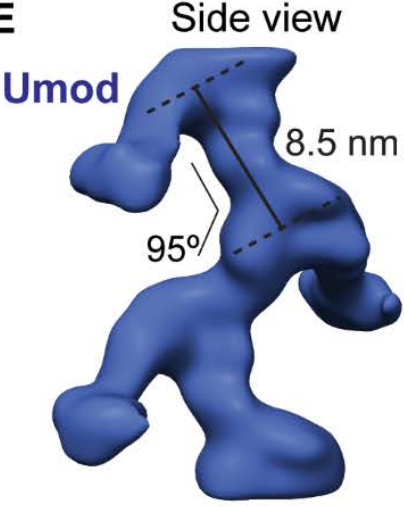

$\mathbf{F}$

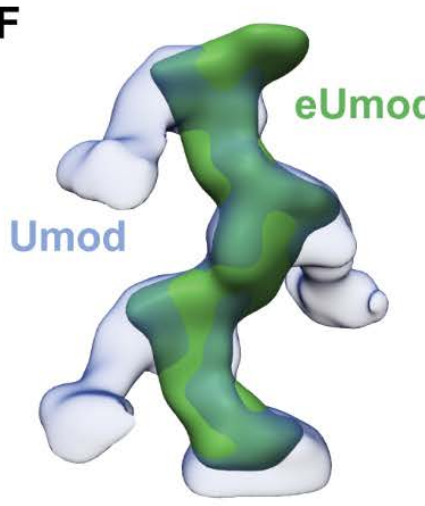

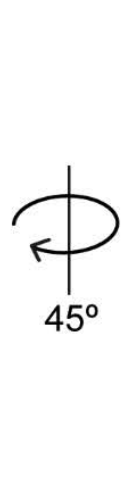
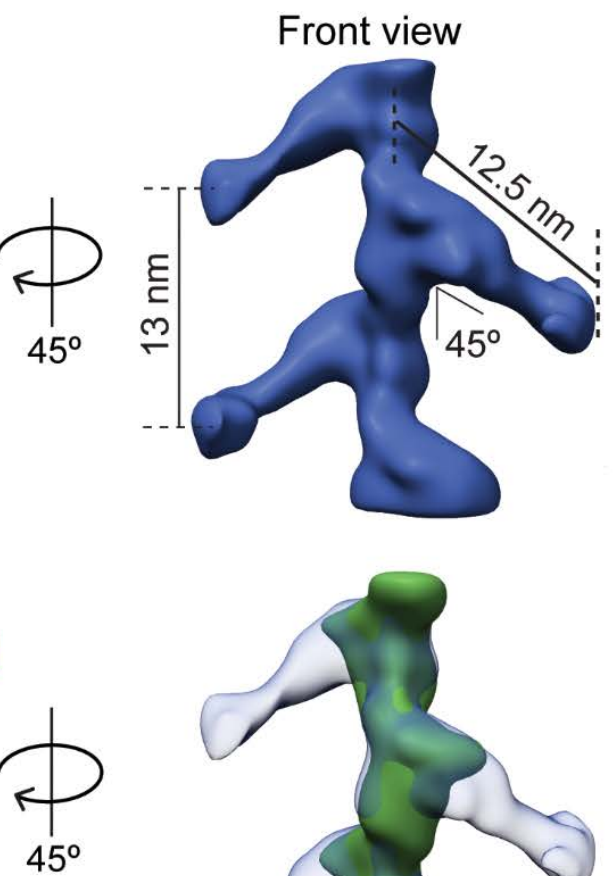

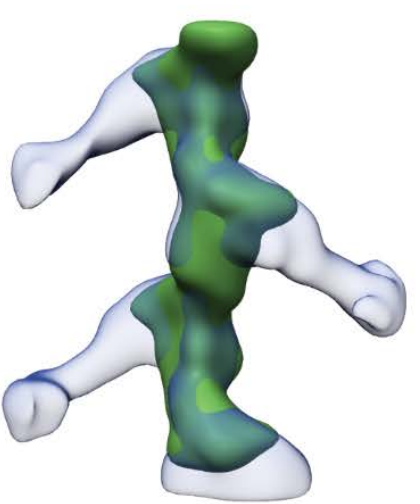

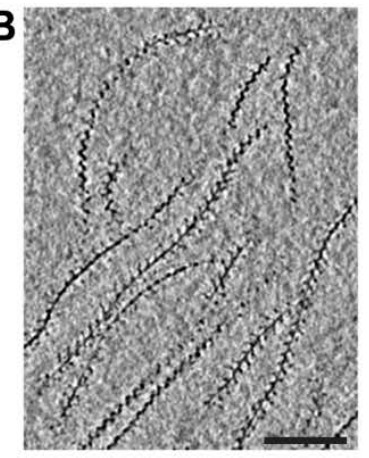

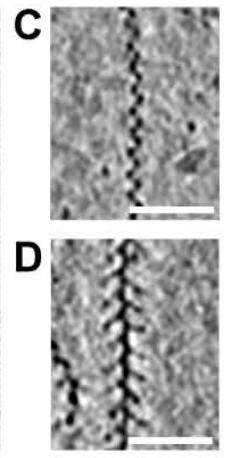

Top view
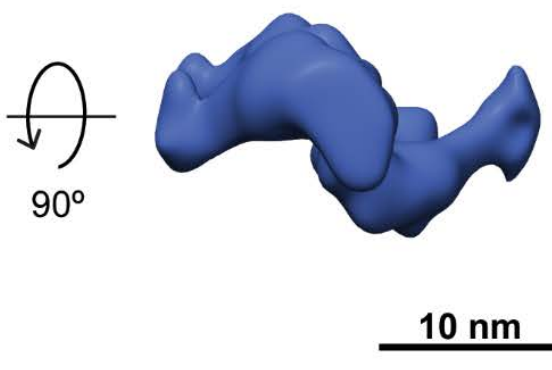

$10 \mathrm{~nm}$

G

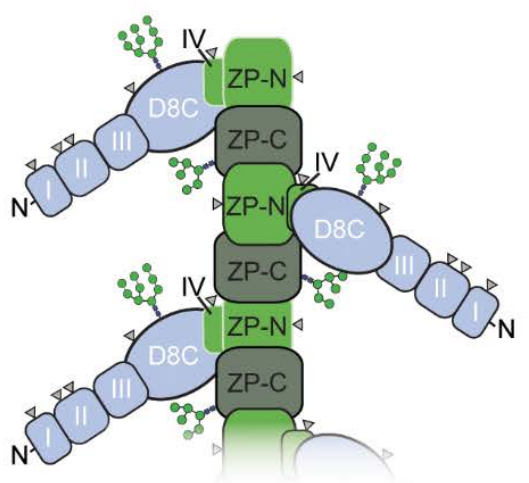

Fig. 1. Umod site-specific glycosylation pattern and filament architecture. (A) Domain organization of mature Umod with four EGF-like domains (I-III, light blue and IV, green), the cysteine-rich D8C domain (blue) and the bipartite ZP module (ZP-N and ZP-C, green) (1). The most abundant N-linked glycan forms identified at each N-glycosylation site are shown schematically (amino acid numbering according to Umod with $\mathrm{N}$-terminal signal peptide). Except for Asn275 and Asn513, all N-glycans were confirmed to be di-, tri-, or tetra-antennary complex type that could be sialylated and/or fucosylated. We observed mixed N-glycan structures at Asn513, comprised of Man6 and, as previously reported, complex type Nglycans (8). The sum of the masses of all identified glycans corresponded well to the average mass difference (20.1 kDa) between glycosylated and deglycosylated Umod. The elastase-digested form of Umod (eUmod = Ser292 through C terminus Phe587) is marked in green and scissors indicate the elastase cleavage site after Ser291. (B to D) CryoET of purified Umod filaments revealed irregular bending and helical parameters. (C) and (D) show magnified views of the two most common orientations, revealing the zigzag core (C) and the lateral arms causing a fishbone-like appearance (D). Shown are $13.8 \mathrm{~nm}$-slices through cryotomograms. Bars, $100 \mathrm{~nm}$ in (B), $50 \mathrm{~nm}$ in (C) and (D). (E) Different orientations of a Umod subtomogram average (surface renderings). The subtomogram average was low-pass filtered to $27 \AA$ to demonstrate the complete three-dimensional architecture. (F) Different orientations (surface renderings, green) of the eUmod subtomogram average. The superposition of eUmod with native Umod (transparent, light blue), low-pass filtered to the same resolution of $27 \AA$, demonstrates the absence of the lateral arms in eUmod. The unfiltered averages can be seen in fig. S9. (G) Proposed alternating ZP-N/ZP-C stacking model of the Umod filament architecture [same orientation as in (F, right)]. The two high-mannose type $\mathrm{N}$-glycan structures in each Umod monomer are indicated by green glycan trees, while complex type $\mathrm{N}$-glycans are shown as gray triangles. 

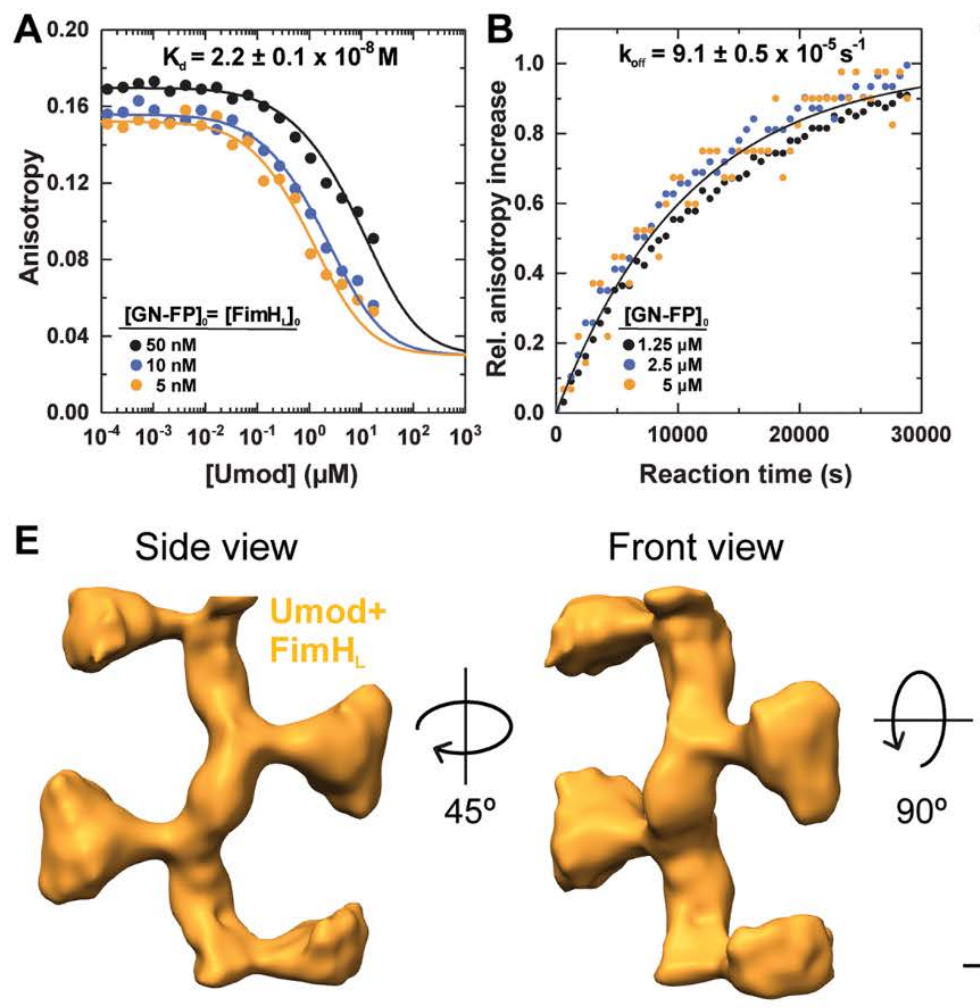

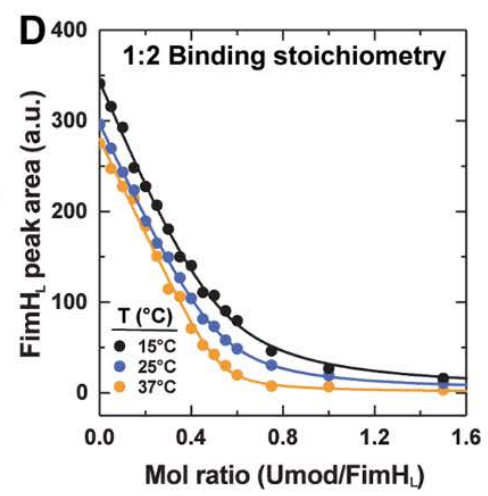

$\mathbf{F}$

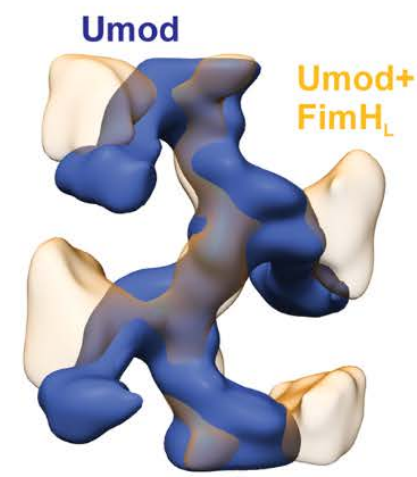

Fig. 2. Two FimH $\mathrm{L}_{\mathrm{L}}$ molecules bind to the high-mannose glycan on the Umod arm. (A and $\left.B\right)$ Thermodynamics and kinetics of Umod binding and release by $\mathrm{FimH}_{\mathrm{L}}$ at $\mathrm{pH} 7.4$ and $25^{\circ} \mathrm{C}$. (A) Competitive equilibrium displacement of the fluorescent mannoside GN-FP $(21,22)$ from $\mathrm{FimH}_{\mathrm{L}}$ with increasing Umod concentrations, recorded via the decrease in GN-FP fluorescence anisotropy. (B) Kinetics of spontaneous dissociation of FimH $\mathrm{L}_{L}$ from Umod, recorded via binding of excess GN-FP to released FimH $\mathrm{H}_{L}$. The obtained first-order kinetics (solid lines) were independent of GN-FP concentration and thus directly monitored dissociation of FimH $\mathrm{L}_{\llcorner}$from Umod $\left(k_{\text {off }}=9.1 \times 10^{-5} \mathrm{~s}^{-1}\right)$. Direct determination of the affinity of full-length FimH for Umod filaments proved to be impossible due to the limited solubility of Umod filaments ( $100 \mu \mathrm{M}$ monomers). (C and D) Titration of FimH $\mathrm{L}_{\mathrm{L}}$ with increasing amounts of Umod filaments to determine the stoichiometry of complex formation at pH 7.4 and different temperatures $\left(15^{\circ} \mathrm{C}, 25^{\circ} \mathrm{C}\right.$, and $\left.37^{\circ} \mathrm{C}\right)$. (C) SEC elution profiles of $10 \mu \mathrm{M} \mathrm{FimH} \mathrm{L}_{\mathrm{L}}$ incubated with different amounts of Umod filaments (Umod monomer concentrations between zero and $20 \mu \mathrm{M}$ ), detected via protein absorbance at $280 \mathrm{~nm}$. (D) Peak area of free $\mathrm{FimH}_{\llcorner}$, plotted against the Umod monomer: Fim $\mathrm{H}_{\llcorner}$ratio, revealing that on average two FimH $H_{L}$ molecules can bind to a single Umod monomer. (E) Different orientations of the subtomogram average (shown as a surface rendering) of Umod filaments that were incubated with a four-fold excess of $\mathrm{FimH}_{\llcorner}$over Umod monomers. Major additional densities are detectable on the filament arms, whereas no extra densities could be identified on the filament core. (F) Overlay of surface renderings of subtomogram averages of native Umod (blue) and Umod incubated with a four-fold excess of $\mathrm{FimH}_{\mathrm{L}}$ (orange, transparent), both low-pass filtered to a resolution of $27 \AA$. The additional densities could accommodate two copies of $\mathrm{FimH}_{\mathrm{L}}$ (fig. S12, D to G). Together, the data indicate that up to two $\mathrm{FimH}_{\mathrm{L}}$ molecules bind to the high-mannose glycosylation site at Asn275 in the D8C domain of the Umod arm. 

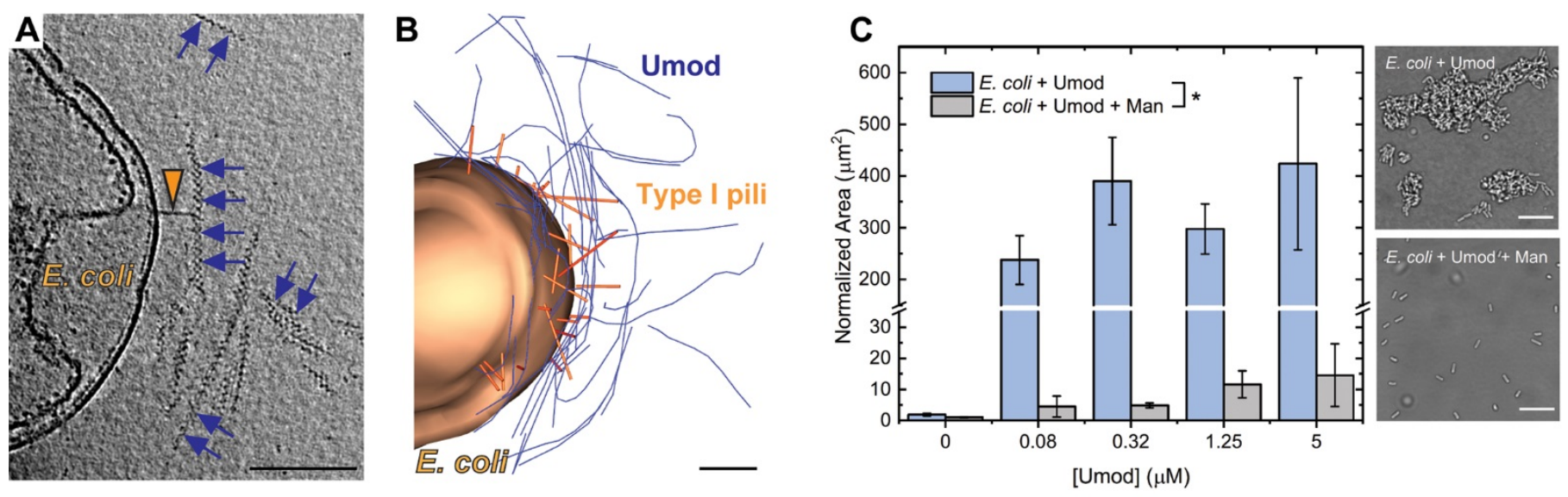
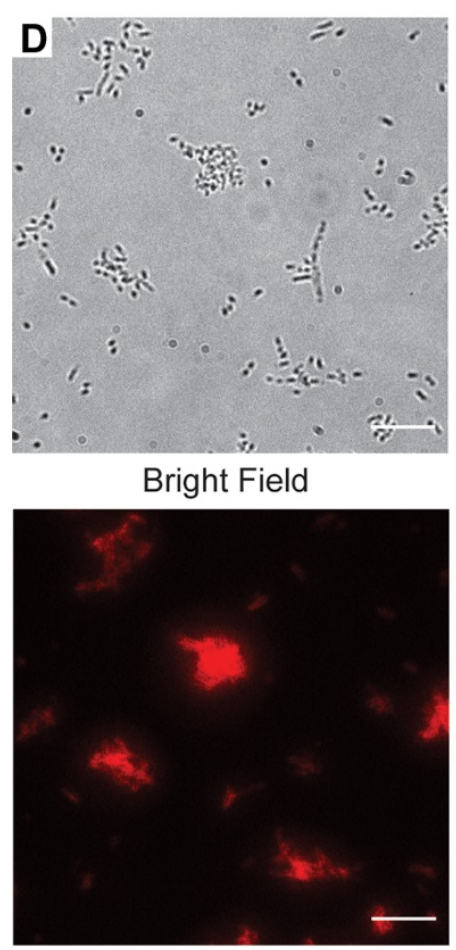

Umod-Alexa647

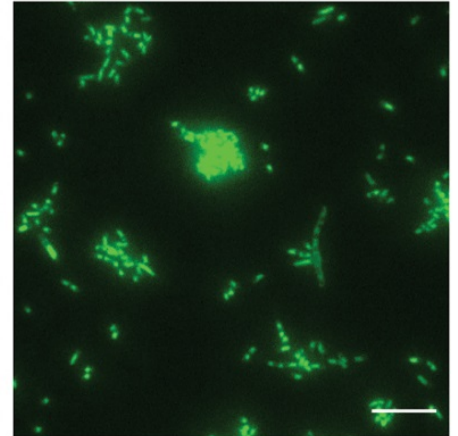

Type 1 piliated E. coli

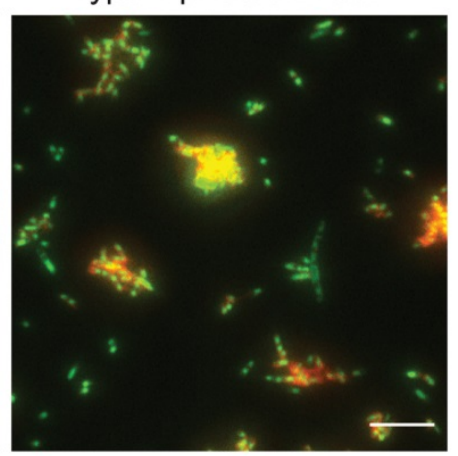

Merge

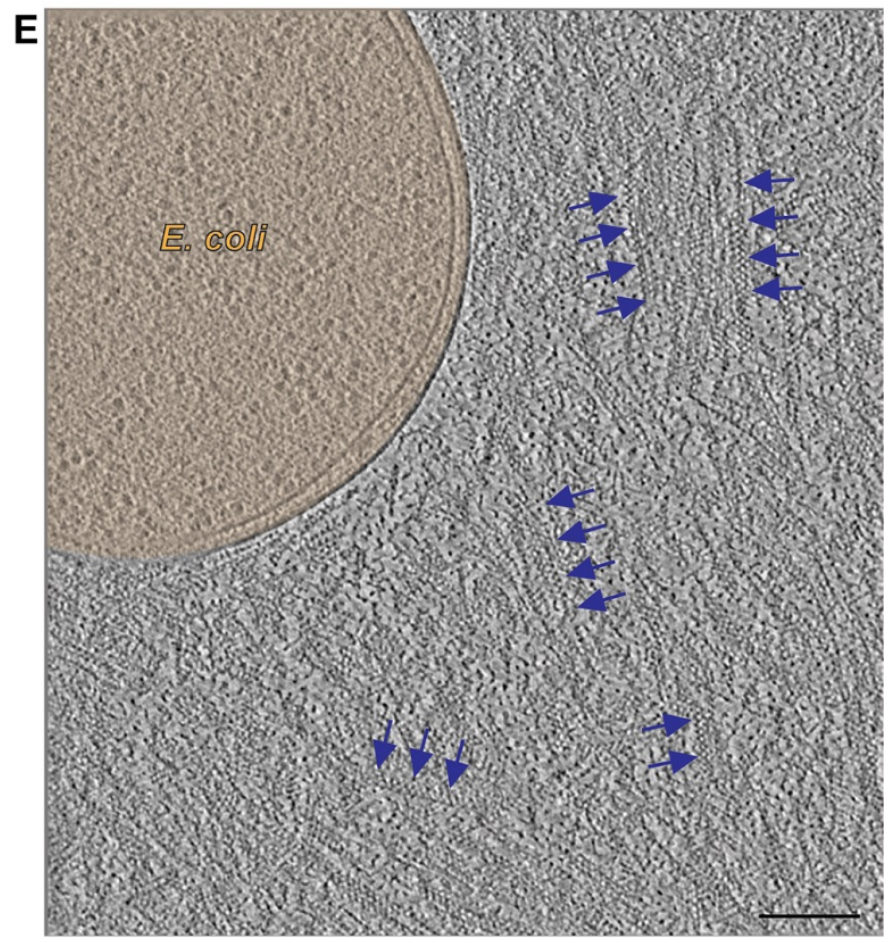

Fig. 3. Umod associates with piliated bacteria and can lead to cell aggregation. (A and B) The type 1-pilus deficient strain E. coli AAEC189 was transformed with the type 1 pilus expression plasmid pSH2, co-incubated with Umod filaments $\left(1 \mu \mathrm{M}\right.$ Umod monomers and $\sim 1.6 \times 10^{9} \mathrm{E}$. coli cells $\left./ \mathrm{ml}\right)$ and imaged by cryoET. Shown is a slice $(8.6 \mathrm{~nm}$ thickness) through a cryotomogram (A) and a segmentation of the same tomogram (B). Piliated bacteria (cell, brown; pili, orange) were always seen in close association with a loose mesh of Umod filaments (blue/arrows). Note the contact site of the pilus tip with the Umod filament. Shown is a partly lysed cell that resulted in higher image quality. See fig. S13, A and B, for more examples. Bars, $100 \mathrm{~nm}$. (C) Quantification of Umod-mediated formation of cell aggregates with light microscopy. The bar graph shows the normalized clump area observed upon incubation (2 hours) at $37^{\circ} \mathrm{C}$ of a constant amount of type 1-piliated $E$. coli HB101 [FimAra] $\left(\sim 10^{8} \mathrm{cells} / \mathrm{mL}\right)$ with different concentrations of Umod filaments (0.08-5.0 $\mu \mathrm{M}$, concentrations refer to the Umod monomer). The presence of 10 mM D-mannose (Man) inhibited aggregation across all Umod concentrations tested. Representative light microscopy images are shown on the right (without and with $10 \mathrm{mM}$ Man). Bars represent the mean of biological triplicates \pm SEM. * $p<0.05$ difference between groups (two-way ANOVA, post-hoc Tukey test). Bars, $10 \mu \mathrm{m}$. (D) Cell aggregates co-localized with Umod filaments. Shown are bright field and TIRF microscopy images of type 1piliated E. coli HB101 [FimAra] cells (excitation at $405 \mathrm{~nm}$, green) that were incubated with fluorescent UmodAlexa647 filaments (red). Bars, $10 \mu \mathrm{m}$. (E) Umod-induced $E$. coli aggregates were plunge-frozen, thinned by cryofocused ion beam milling, and imaged by cryoET (shown is an $8.6 \mathrm{~nm}$ tomographic slice). E. coli cells (brown) were always seen embedded in a highly dense meshwork of Umod filaments (blue arrows). For further examples see fig. S19. Bar, $100 \mathrm{~nm}$. 

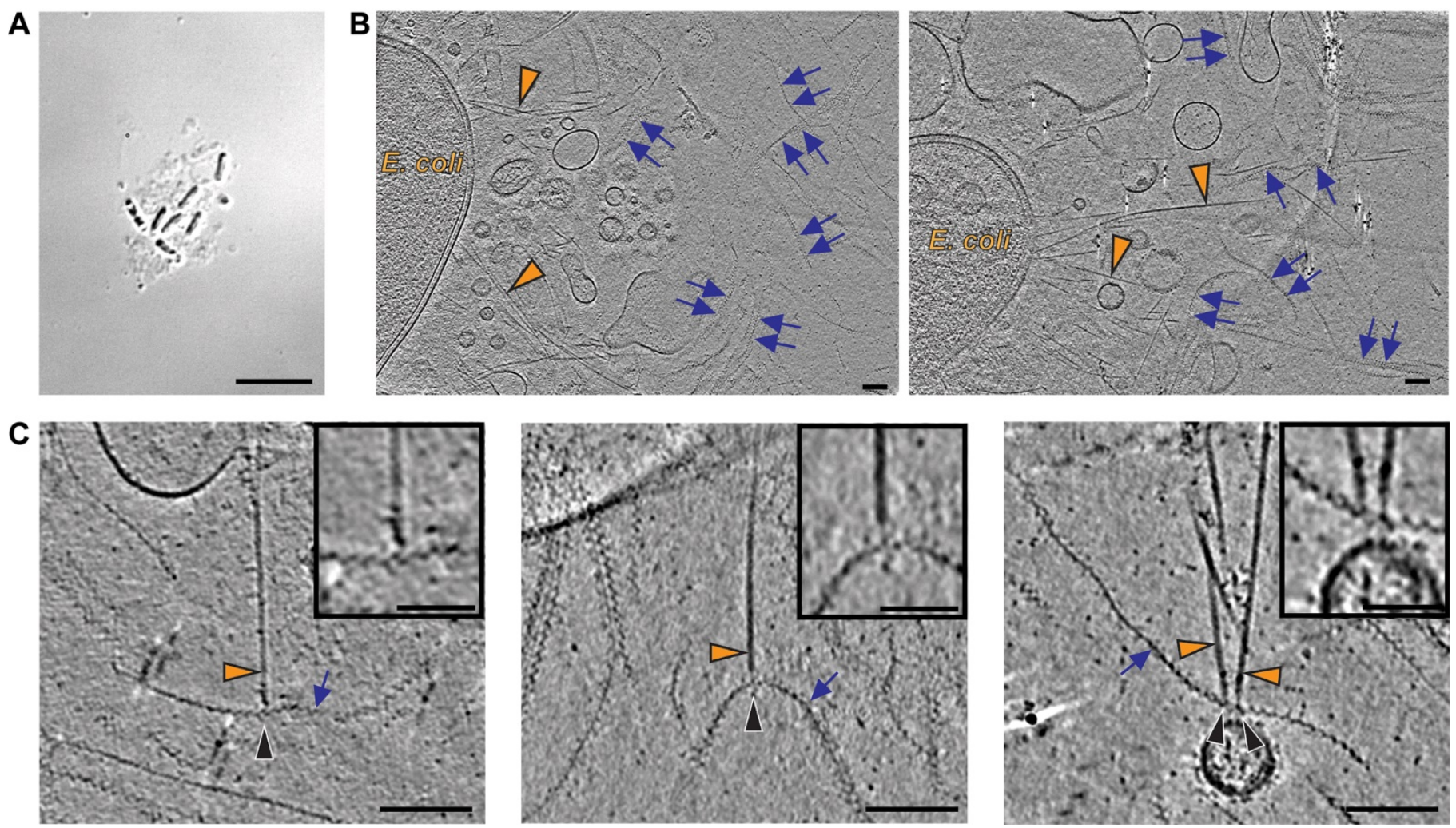

Fig. 4. Urine from UTI patients shows cell aggregation and pilus-mediated Umod association with bacterial cells. (A) DIC light microscopy imaging of urine from a patient with acute $E$. coli UTI revealed clustered bacteria. Bar, $10 \mu \mathrm{m}$. (B) CryoET imaging of urine from the same patient showed piliated (orange arrowheads) bacterial cells (labeled "E. coli"). All analyzed cells ( $n=27$ tomograms/cells, shown are two representative examples) were surrounded by filaments with the typical Umod appearance (blue arrows). Bars, $100 \mathrm{~nm}$. (C) The cryotomograms revealed multiple contact sites (black arrowheads) between pili (orange arrowheads) and Umod (blue arrows). Bars, $100 \mathrm{~nm}$. Magnified views in insets. Bars, $50 \mathrm{~nm}$. 